\title{
Changing Health Perceptions Among the Elderly
}

There has been an increasing emphasis on patient assessment in recent literature, including a number of articles and editorials in the Journal. The importance of reassessment, however, has received only scant attention. Although few would disagree that older patients' health can change rapidly over time, variability in self-reported changes in health can be unpredictable, representing a challenge to treatment.

The patient-doctor relationship is often the critical element in assessment. The success of the relationship is determined, at least in part, by the physician's knowledge of how individual patients view their health. Being attuned to such changes becomes more important with chronically ill older patients who are seen more often and for longer time periods. Patients' perceptions of their health and treatment are likely to vary on the basis of real changes in their health over time, as well as for other reasons such as the faulty exchange of information. For example, the knowledge and authority attributed to the physician can produce a one-sided communication process in which the doctor assumes responsibility for identifying information relevant to treatment. Thus, older patients can too easily become passive participants, relying instead on the doctor's specialized knowledge and expertise. ${ }^{1}$ Also easily overlooked are patients' personal and family concerns, their health beliefs, and changes in general attitudes toward life, all of which gain importance during extended periods of illness and become more likely to influence health perceptions. ${ }^{2}$

We interviewed 56 patients three times during their first 6 months following referral to a specialized geriatric outpatient clinic, and found little consistency in their self-health perceptions before and after their initial visit, ${ }^{3}$ and even less when we reinterviewed several months later $(P<.01)$. Moreover, there was no pattern or objective basis for many of the changes, including changes in the ways they dealt with fairly critical health information.

We also found that the oldest patients approached each clinic visit with cautious uncertainty about their health if not denial of problems. This uncertainty arose from fears of functional decline and its impact on their autonomy and daily lives-particularly the potential threat of institutionalization. Overall uncertainty may encompass the much larger issue of the impact of chronic illness on the daily lives of the elderly. Adaptive strategies that were previously successful with acute problems may not work with chronic illness. The failure of these strategies can cause uncertainty about how to deal with the illness and how to interact with physicians.

Thus, current research suggests the potential difficulty of predicting future patterns of change based on health-related judgments made early in treatment. Although most physicians would be quick to say that the patient always gets a "new look" at each visit, much weight is often assigned to the chart-especially in clinical settings where patients are seen by different physicians. In the case of the geriatric patient, the medical record is, at best, incomplete.

Among the implications for geriatric practice are the obvious value of updated information from patients about their health and treatment expectations, and the need for greater sensitivity to the impact of changing perceptions on compliance and on general health behavior.

TOM HICKEY Professor and Director Health Gerontology Program School of Public Health

The University of Michigan

\section{REFERENCES}

1. Haug MR: Doctor-patient relationships and the older patient. J Gerontol 34:852, 1979

2. Breslau L: Problems of maintaining a therapeutic viewpoint, in Haug MR (ed): Elderly Patients and Their Doctors. New York, Springer, 1981, p 119

3. Hickey $\mathrm{T}$, Rakowski W: Consistency in patient's health and treatment expectations at a geriatric clinic. J Am Geriatr Soc 29:278, 1981 